\title{
Chloroform fraction of Scutellaria barbata D. Don promotes apoptosis and suppresses proliferation in human colon cancer cells
}

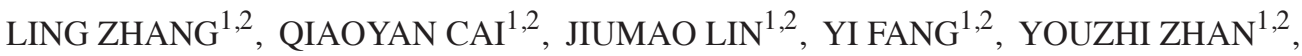 \\ ALING SHEN $^{1,2}$, LIHUI WEI ${ }^{1,2}$, LILI WANG ${ }^{1,2}$ and JUN PENG ${ }^{1,2}$ \\ ${ }^{1}$ Academy of Integrative Medicine and ${ }^{2}$ Fujian Key Laboratory of Integrative Medicine on Geriatrics, \\ Fujian University of Traditional Chinese Medicine, Fuzhou, Fujian 350122, P.R. China
}

Received June 3, 2013; Accepted December 2, 2013

DOI: $10.3892 / \mathrm{mmr} .2013 .1864$

\begin{abstract}
Scutellaria barbata D. Don (SB) has long been used as a major component in numerous Chinese medical formulas to clinically treat various types of cancer. Previously, we reported that the extracts of SB were able to suppress colon cancer growth in vivo and in vitro, possibly by inducing cancer cell apoptosis and inhibiting cell proliferation and tumor angiogenesis. However, the anticancer mechanisms of its bioactive ingredients remain largely unclear. In the present study, using three human colon cancer cell lines SW620, HT-29 and HCT-8, the antitumor effect of different solvent fractions of SB were evaluated and the potential underlying molecular mechanisms were investigated. Using an 3-(4, 5-dimethylthiazol-2-yl)-2,5-diphenyltetrazolium bromide assay, it was revealed that the chloroform fraction of SB (ECSB) exhibited the most potent inhibitory effect on the growth of all three colon cancer cell lines and SW620 cells exhibited the most sensitive response to ECSB treatment $\left(\mathrm{IC}_{50}=65 \mu \mathrm{g} / \mathrm{ml}\right)$. In addition, by performing fluorescence-activated cell sorting, transmission electron microscopy and colony formation assays, it was observed that ECSB significantly induced apoptosis and inhibited proliferation in SW620 cells in a dose-dependent manner. Furthermore, ECSB treatment resulted in the upregulation of the pro-apoptotic Bax/Bcl-2 ratio and a decrease in the expression of the pro-proliferative cyclin D1 and cyclin-dependent
\end{abstract}

Correspondence to: Dr Jun Peng, Academy of Integrative Medicine, Fujian University of Traditional Chinese Medicine, 1 Huatuo Road, Fuzhou, Fujian 350122, P.R. China

E-mail: pjunlab@hotmail.com

Abbreviations: CRC, colorectal cancer; SB, Scutellaria barbata D. Don; ECSB, chloroform fraction of Scutellaria barbata D. Don; EPESB, petroleum ether fraction of Scutellaria barbata D. Don; EEASB, ethyl acetate fraction of Scutellaria barbata D. Don; ENSB, N-butanol fraction of Scutellaria barbata D. Don; TCM, traditional Chinese medicine; TEM, transmission electron microscopy; MTT, 3-(4, 5-dimethylthiazol-2-yl)-2,5-diphenyltetrazolium bromide

Key words: herbal medicine, Scutellaria barbata D. Don, polar fractions, colorectal cancer, apoptosis, proliferation kinase 4. The results from the present study may provide a scientific foundation for the development of novel anticancer agents from the bioactive ingredients in the ECSB.

\section{Introduction}

Colorectal cancer (CRC) is one of the most serious malignancies with its incidence and mortality increasing annually (1). Although surgical resection offers the greatest prognosis, a substantial portion of CRC patients already present with metastatic disease at the time of diagnosis $(2,3)$. Additionally, surgery is not always able to extirpate the recurrence of advanced CRC (4). Therefore, chemotherapy remains one of the major non-surgical therapeutic approaches for patients with advanced CRC. However, the efficacy and safety of the chemotherapies which are currently used remains a challenge due to severe toxicity, multidrug resistance and other side effects (5-7). Faced with these major problems, the development of novel anticancer agents is urgently required. Traditional Chinese medicine (TCM), dating back thousands of years, is important in the treatment of various diseases, including cancer (8-10). Clinical practice has also demonstrated that numerous TCMs are effective for the treatment of CRC (11).

Scutellaria barbata D. Don (SB) is a medicinal herb widely distributed in northeast Asia. As a well known traditional Chinese folk medicine, it has long been used to clinically treat various types of cancer (12-16). In particular, our previous studies demonstrated that the extracts of SB were able to suppress colon cancer growth in vivo and in vitro, possibly by inducing cancer cell apoptosis and inhibiting cell proliferation and tumor angiogenesis (16-18). However, the anticancer mechanisms of its bioactive ingredients are largely unclear. In the present study, using three human colon cancer cell lines SW620, HT-29 and HCT-8, the antitumor effect of different polar fractions of SB were evaluated and the potential underlying molecular mechanisms were investigated. It was revealed that the chloroform fraction of SB (ECSB) exhibited the most potent inhibitory effect on the growth of all three colon cancer cell lines and SW620 cells exhibited the most sensitive response to ECSB treatment. In addition, ECSB promoted apoptosis via the upregulation of the pro-apoptotic $\mathrm{Bax} / \mathrm{Bcl}-2$ ratio and inhibited proliferation by suppressing the expression of the pro-proliferative cyclin D1 and cyclin-dependent kinase 4 (CDK4) in SW620 cells. 


\section{Materials and methods}

Materials and reagents. RPMI-1640 medium, KGML-15 SY medium, fetal bovine serum (FBS), penicillin-streptomycin and trypsin-EDTA were obtained from Hyclone (Carlsbad, CA, USA). TRIzol reagent and SuperScript II reverse transcriptase were purchased from Invitrogen Life Technologies (Grand Island, NY, USA). Anti-Bcl-2, Bax, Cyclin D1, CDK4 and $\beta$-actin antibodies, and horseradish peroxidase (HRP)-conjugated secondary antibodies were obtained from Cell Signaling Technology (Beverly, MA, USA). A fluorescein isothiocyanate (FITC)-conjugated Annexin V apoptosis detection kit was provided by Becton-Dickinson (San Jose, CA, USA). All the other chemicals, unless otherwise stated, were obtained from Sigma-Aldrich (St. Louis, MO, USA).

Preparation of the SB extract. The herb was obtained from the Guo Yi Tang Chinese Herbal medicine store (Fujian, China). SB $(500 \mathrm{~g})$ was extracted three times with $5,000 \mathrm{ml}$ of $85 \%$ ethanol using a refluxing method and filtered. The solvent was fractionated by a series of solvents, including petroleum ether, chloroform, ethyl acetate and N-butanol, to obtain the petroleum ether fraction of SB (EPESB), ECSB, ethyl acetate fraction of SB (EEASB) and the N-butanol fraction of SB (ENBSB). These fractions were then evaporated on a rotary evaporator. They were all dissolved in $100 \%$ dimethylsulfoxide (DMSO) to a stock concentration of $200 \mathrm{mg} / \mathrm{ml}$ and stored at $-20^{\circ} \mathrm{C}$. The final concentration of DMSO in the medium for all the experiments was $\leq 0.25 \%$.

Cell culture. Human carcinoma SW620, HT-29 and HCT-8 cells were obtained from the Cell Bank of the Chinese Academy of Sciences (Shanghai, China). SW620 cells were grown in L-15 medium and HT-29 and HCT-8 cells were cultured in RPMI-1640 medium, supplemented with $10 \%$ fetal bovine serum, $100 \mathrm{U} / \mathrm{ml}$ penicillin and $100 \mathrm{mg} / \mathrm{ml}$ streptomycin at $37^{\circ} \mathrm{C}$ in $5 \% \mathrm{CO}_{2}$ humidified air.

Cell viability using an 3-(4, 5-dimethylthiazol-2-yl)-2,5-diphenyltetrazolium bromide (MTT) assay. Cell viability was assessed by an MTT colorimetric assay. SW620, HT-29 and HCT- 8 cells were seeded into 96-well plates at a density of $5 \times 10^{5}, 3 \times 10^{5}$ and $1 \times 10^{5}$ cells $/ \mathrm{ml}$ in $100 \mu$ l of medium, respectively. The cells were treated with serial concentrations of fractions of SB for $24 \mathrm{~h}$. Following treatment, $100 \mu \mathrm{l}$ of MTT $(0.5 \mathrm{mg} / \mathrm{ml}$ in PBS) was added to each well and the cells were incubated at $37^{\circ} \mathrm{C}$ for $4 \mathrm{~h}$. The medium was removed and the purple-blue MTT formazan precipitate was dissolved in $100 \mu \mathrm{l}$ of DMSO. The absorbance was measured at $570 \mathrm{~nm}$ using an ELISA reader (model ELX800; BioTek, Winooski, VT, USA). The cell viability was determined using the formula: Cell viability $(\%)=$ sample optical density $(\mathrm{OD}) /$ control OD x 100 .

Detection of apoptosis by flow cytometric analysis with Annexin V/propidium iodide (PI) staining. Following starvation for $12 \mathrm{~h}, \mathrm{SW} 620$ cells were incubated for $24 \mathrm{~h}$ in various concentrations of ECSB. Apoptosis of SW620 cells was determined by flow cytometric analysis using a fluorescence-activated cell sorting (FACS) caliber (Becton-Dickinson) and an
Annexin V-FITC/PI kit (Becton-Dickinson). The procedure was performed according to the manufacturer's instructions. Annexin V-positivity and PI-negativity indicated the presence of early apoptotic cells, while Annexin V-positivity and PI-positivity indicated the presence of late apoptotic cells.

Observation of ultrastructural characteristics by transmission electron microscopy (TEM). Cells were fixed with $1.5 \%$ paraformaldehyde and 3\% glutaraldehyde in $0.1 \mathrm{~mol} / 1$ sodium cacodylate buffer ( $\mathrm{pH} 7.2-7.4)$ at $4^{\circ} \mathrm{C}$ for $24 \mathrm{~h}$. The cell suspensions were then rinsed twice with phosphate-buffered saline (PBS) and post-fixed with $1 \%$ osmic acid in $0.1 \mathrm{~mol} / 1$ sodium cacodylate buffer for $2 \mathrm{~h}$. The cells were dehydrated in a graded series of alcohol and embedded with epoxy resin 618 . Ultrathin sections $(80 \mathrm{~nm})$ were mounted on the copper wire mesh grids, air-dried, stained with $2.0 \%$ uranyl acetate for $15 \mathrm{~min}$ and counterstained with lead citrate for $15 \mathrm{~min}$. The sections were examined and images were captured using a Hitachi 7650 electron microscope (Tokyo, Japan).

Colony formation. SW620 cells were seeded into 6-well plates at a density of $5 \times 10^{5}$ cells $/ \mathrm{ml}$ in $2 \mathrm{ml}$ medium. Following treatment with various concentrations of ECSB for $48 \mathrm{~h}$, cells were harvested and diluted in $2 \mathrm{ml}$ fresh medium without ECSB, and then each well was reseeded at a density of 1,000 cells per well. The medium was replaced with fresh medium every three days. Following 14 days, cells were fixed with $10 \%$ formaldehyde, stained with $0.01 \%$ crystal violet and counted. The cell survival rate was calculated by normalizing the survival rate of the control cells to $100 \%$.

Reverse transcription-polymerase chain reaction (RT-PCR) analysis. SW620 cells were exposed to various concentrations of ECSB for $48 \mathrm{~h}$ and then total RNA was isolated with TRIzol reagent (Invitrogen Life Technologies). First-strand cDNA was generated via reverse transcription of $2 \mu \mathrm{g}$ total RNA using Oligo(dT) primer and SuperScript II reverse transcriptase according to the manufacturer's instructions. The obtained cDNA was used to determine the mRNA levels of Bax, Bcl-2, Cyclin D1 and CDK4 by PCR with TaqDNA polymerase (Fermentas, Vilnius, Lithuania). GAPDH was used as an internal control.

Western blot analysis. SW620 cells were treated with various concentrations of ECSB for $48 \mathrm{~h}$. Adherent and floating cells were harvested and rinsed three times with PBS buffer. The cells were lysed with radioimmunoprecipitation assay lysis buffer (Pierce Chemical Co., Rockford, Illinois, USA) containing phenylmethanesulfonyl fluoride and extracts were quantified using the bicinchoninic acid protein assay (Pierce Chemical Co.) The proteins $(30 \mu \mathrm{g})$ were separated by $12 \%$ SDS-PAGE gels and transferred onto polyvinylidene fluoride membranes (Millipore Corporation, Billerica, MA, USA). The membranes were inhibited with 5\% skimmed milk and probed with primary antibodies against Bax, Bcl-2, cyclin D1, CDK4 and $\beta$-actin $(1: 1,000)$ overnight at $4^{\circ} \mathrm{C}$. The membranes were rinsed three times with Tris-buffered saline with Tween-20 (TBST) and then the appropriate HRP-conjugated secondary antibodies were diluted at 1:5,000 in blocking solutions for $1 \mathrm{~h}$ at room temperature. Following washing again in TBST, the 
A

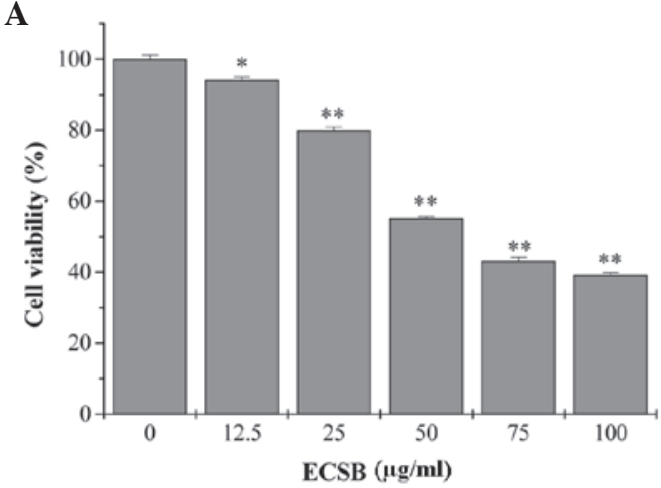

C

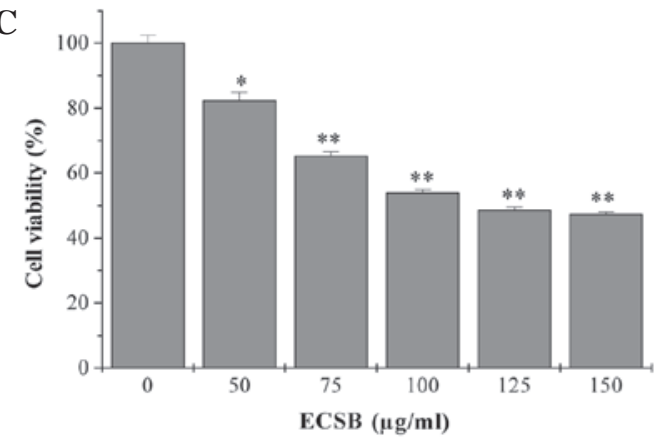

$\mathbf{E}$

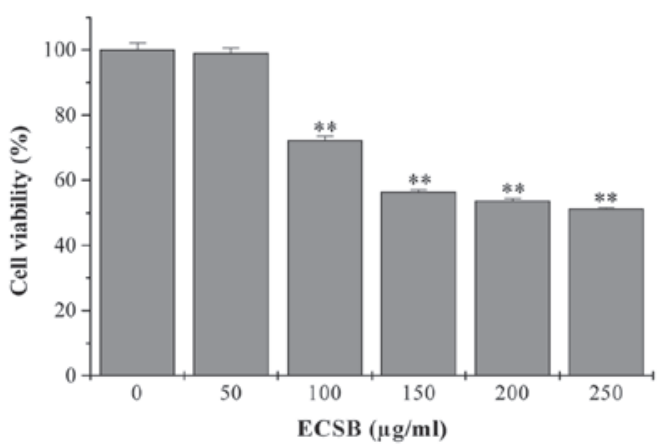

B
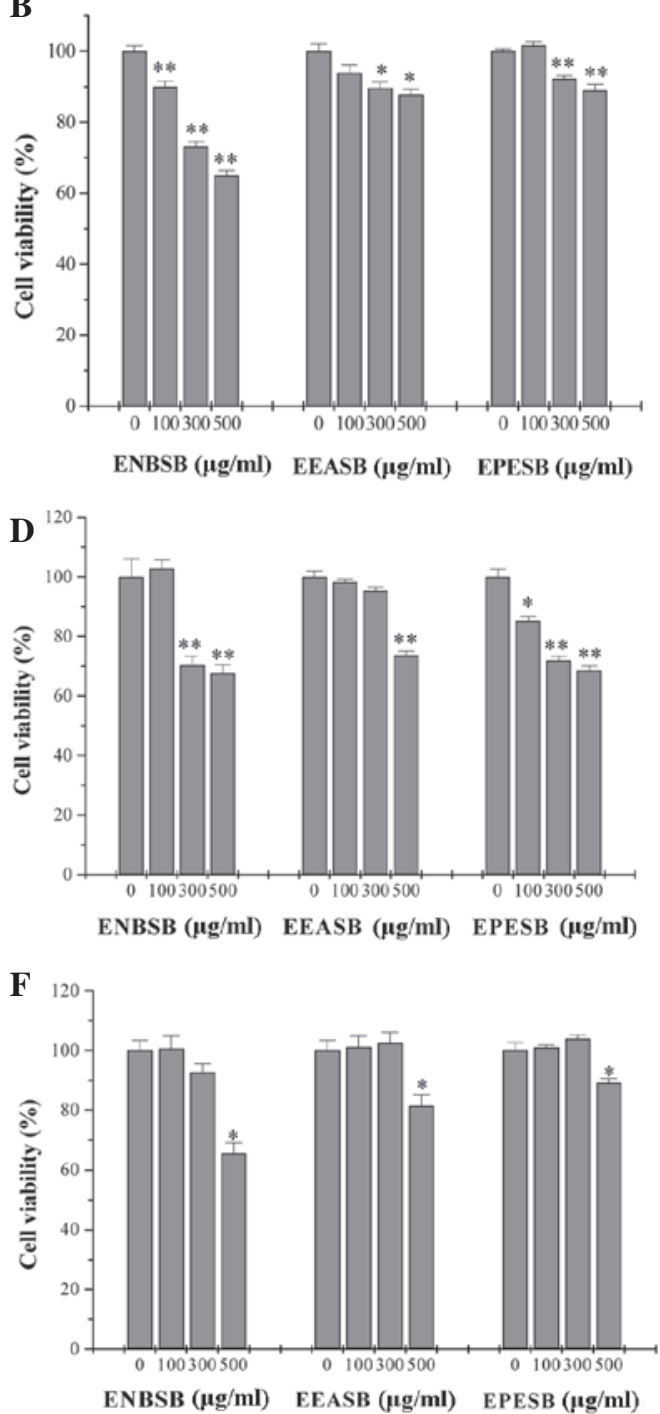

Figure 1. Effect of different polar fractions of SB on the viability of human colon cancer cells. (A and B) SW620, (C and D) HT-29 and (E and F) HCT-8 cells were treated with ECSB, ENSB, EEASB or EPESB for $24 \mathrm{~h}$, respectively. Cell viability was determined by the MTT assay. The data were normalized to the viability of the untreated control cells $(100 \%)$. Data are expressed as the mean \pm standard deviation (error bars) from at least three independent experiments. ${ }^{*} \mathrm{P}<0.05 ;{ }^{* *} \mathrm{P}<0.01$, versus untreated control cells. SB, Scutellaria barbata D. Don; ECSB, chloroform fraction of SB; EPESB, petroleum ether fraction of SB; EEASB, ethyl acetate fraction of SB; ENSB, N-butanol fraction of SB.

membranes were detected by enhanced chemiluminescence. $\beta$-actin was used as an internal control.

Statistical analysis. Data were analyzed using the SPSS package for Windows (version 13.0; SPSS Inc., Chicago, IL, USA). The quantitative data are expressed as the mean \pm standard deviation. Statistical analysis of the data was performed using Student's t-test. $\mathrm{P}<0.05$ was considered to indicate a statistically significant difference.

\section{Results and Discussion}

ECSB exhibits the most potent inhibitory effect on the growth of colon cancer cells. By performing an MTT assay in three human colon cancer cell lines, SW620, HT-29 and HCT-8, the in vitro anticancer effect of different fractions of $\mathrm{SB}$, including EPESB, ECSB, EEASB and ENSB, was compared. As shown in Fig. 1, all the fractions inhibited the viability of three cancer cells in a dose-dependent manner. In particular, ECSB exhibited the most potent antitumor activity in SW620 cells, with an $\mathrm{IC}_{50}$ of $65 \mu \mathrm{g} / \mathrm{ml}$. To further examine the mode of action of ECSB, SW620 cells were selected for the following study.

ECSB promotes apoptosis and inhibits the proliferation of SW620 cells. Apoptosis eliminates excess, redundant, abnormal cells in animals and thus is crucial for animal development and tissue homeostasis. Disturbed regulation of this vital process represents a major causative factor in tumorigenesis (19). Therefore, in order to determine the mechanism of the growth suppressive activity of ECSB, its effect on apoptosis in SW620 cells was examined. The in vitro cell apoptosis was assessed via Annexin V/PI staining followed by FACS analysis. As shown in Fig. 2, the percentage of cells undergoing either early apoptosis or late apoptosis following treatment with $0,25,50$ and $75 \mu \mathrm{g} / \mathrm{ml}$ ECSB was $6.80,11.54$, 33.53 and $65.12 \%$, respectively $(\mathrm{P}<0.05)$, suggesting that ECSB 
$\mathbf{A}$
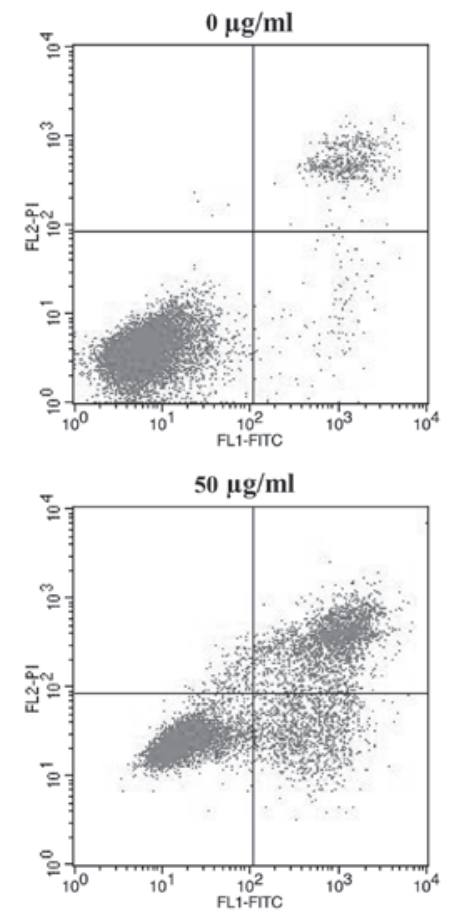

B
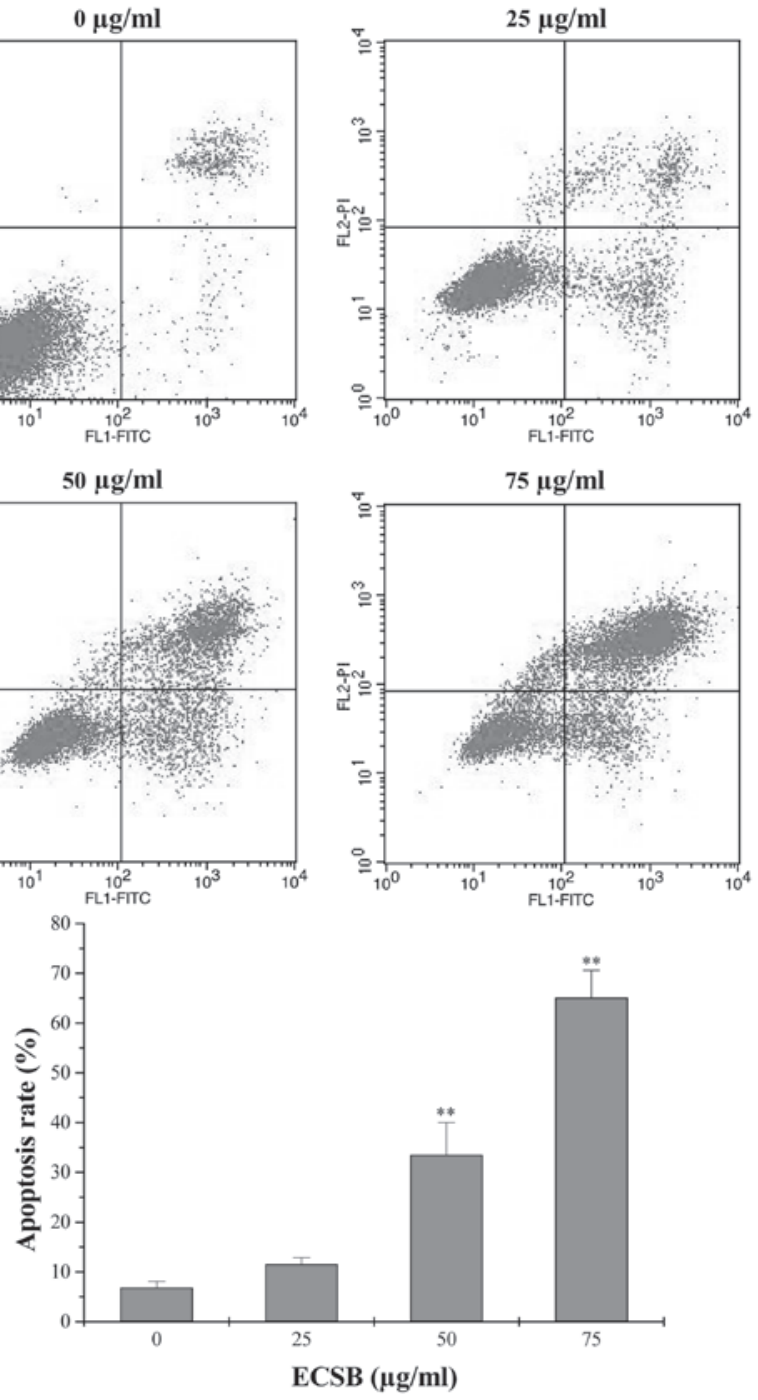

Figure 2. Effect of ECSB on the apoptosis of SW620 cells. (A) Following starvation for $12 \mathrm{~h}, \mathrm{SW} 620$ cells were incubated for $24 \mathrm{~h}$ at various concentrations of ECSB and then cells were collected and stained with Annexin V/PI followed by FACS analysis. (B) Quantification of FACS analysis. The data are expressed as the mean \pm standard deviation (error bars) from three independent experiments. ${ }^{* *} \mathrm{P}<0.01$, vs. the untreated control cells. ECSB, chloroform fraction of Scutellaria barbata D. Don; FACS, fluorescence-activated cell sorting; PI, propium iodide; FITC, fluorescein isothiocyanate.

treatment significantly induced apoptosis in SW620 cells in a dose-dependent manner.

In order to verify these results, TEM was used to observe ultrastructural changes in SW620 cells following ECSB treatment. As shown in Fig. 3, untreated cells exhibited a normal ultrastructure, including numerous microvilli on the cell surface, evenly distributed chromatin, a large nucleolus in the nucleus and numerous large mitochondria in the cytoplasm. By contrast, following treatment with ECSB, SW620 cells underwent significant ultrastructural changes that represented the typical morphological features of apoptosis, including a low nucleus/cytoplasm ratio, chromatin margination and condensation, formation of vacuoles in the mitochondria, formation of secondary lysosomes, loss of cellular microvilli and mitochondrial cristae. These data further demonstrated the pro-apoptotic activity of ECSB.

Cancer cells are also characterized by uncontrolled proliferation, therefore, inhibiting the excessive proliferation of tumor cells is one of the key approaches for the development of anticancer drugs. In order to determine the effect of
ECSB on the proliferation of cancer cells, the survival rate in ECSB-treated SW620 cells was evaluated using a colony formation assay. As shown in Fig. 4, treatment with 25, 50 and $75 \mu \mathrm{g} / \mathrm{ml}$ ECSB for $48 \mathrm{~h}$, respectively, reduced the survival rate of SW620 cells to 93.42, 54.82 and $5.85 \%$, as compared with the untreated control cells $(\mathrm{P}<0.05)$, suggesting that ECSB suppressed the proliferation of colon cancer cells in a dose-dependent manner.

ECSB regulated the expression of Bax, Bcl-2, cyclin D1 and $C D K 4$. Bcl-2 family proteins are key regulators of apoptosis, functioning as either suppressors, including Bcl-2, or promoters, including Bax (20). Tissue homeostasis is maintained by controlling the ratio of active anti- and pro-apoptotic Bcl-2 family proteins. A higher Bcl-2/Bax ratio caused by aberrant expression of the proteins is commonly found in various types of cancer, which not only confers a survival advantage to the cancer cells, but also results in drug resistance. Eukaryotic cell proliferation is primarily regulated by the cell cycle. G1/S transition is one of the main checkpoints of the cell cycle 


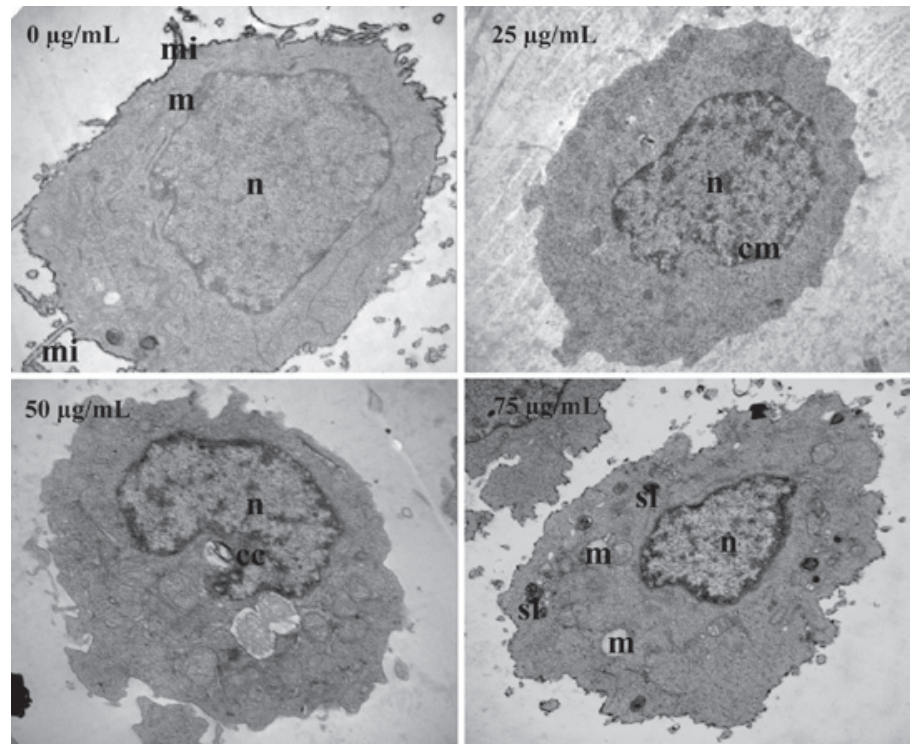

Figure 3. Effect of ECSB on the ultrastructural changes of SW620 cells. Cells were treated with the indicated concentrations of ECSB for $48 \mathrm{~h}$ and ultrastructural changes were observed using transmission electron microscopy. Images were captured at a magnification of x15,000. Images are representative of three independent experiments. $\mathrm{n}$, nucleus; $\mathrm{m}$, mitochondria; mi, microvilli; cm, chromatin margination; cc, chromatin condensation; sl, secondary lysosome; ECSB, chloroform fraction of Scutellaria barbata D. Don.

A

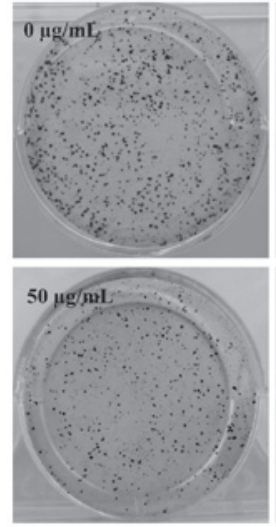

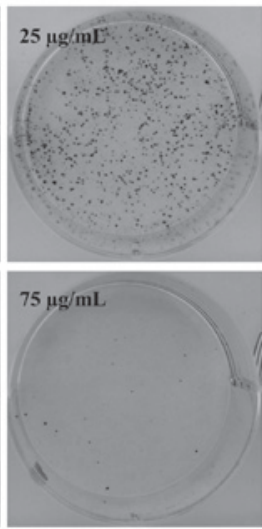

\section{B}

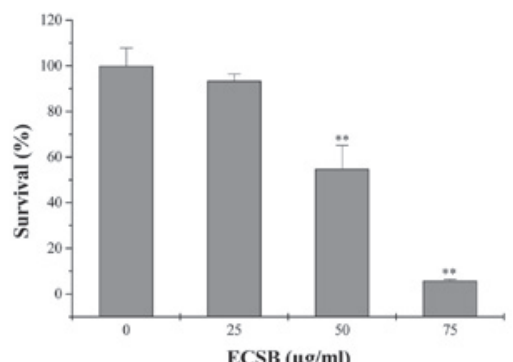

$\operatorname{ECSB}(\mu \mathrm{g} / \mathrm{ml})$

Figure 4. Effect of ECSB on the proliferation of SW620 cells. Cells were treated with the indicated concentrations of ECSB for $48 \mathrm{~h}$. (A) Cell survival rate was determined by a colony formation assay. (B) Quantification of colony formation. The data are expressed as the mean \pm standard deviation (error bars) from three independent experiments. ${ }^{* *} \mathrm{P}<0.01$, vs. the untreated control cells. ECSB, chloroform fraction of Scutellaria barbata D. Don.

A

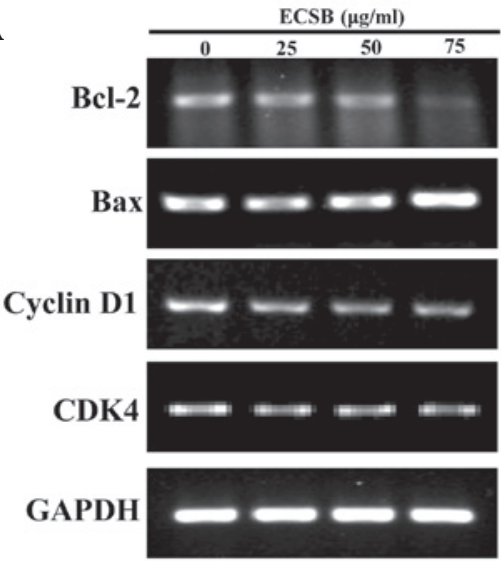

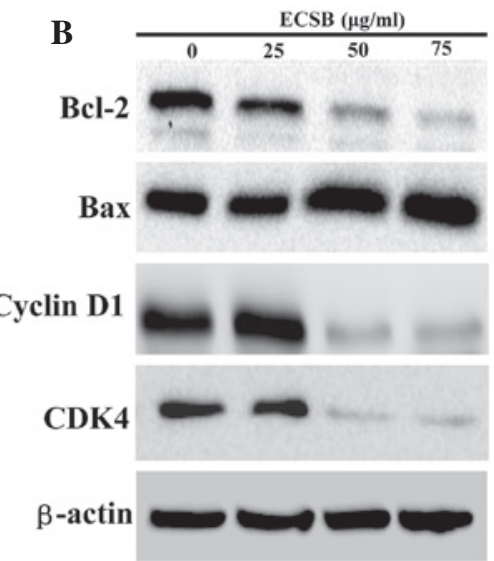

Figure 5. Effect of ECSB on the expression of Bcl-2, Bax, cyclin D1 and CDK4 in SW620 cells. Cells were treated with the indicated concentrations of ECSB for 48 h. (A) mRNA expression of Bcl-2, Bax, Cyclin D1 and CDK4 were evaluated by RT-PCR. (B) Protein expression levels of Bcl-2, Bax, cyclin D1 and CDK4 were determined by western blotting. GAPDH and $\beta$-actin were used as the internal controls for the RT-PCR or western blotting, respectively. Images are representative of three independent experiments. ECSB, chloroform fraction of Scutellaria barbata D. Don; CDK4, cyclin dependent kinase 4; RT-PCR, reverse transcription-polymerase chain reaction. 
and is responsible for the initiation and completion of DNA replication. G1/S progression is regulated by cyclin D1, which exerts its function via forming an active complex with its CDK major catalytic partners (CDK4/6) $(21,22)$. An unchecked or hyperactivated cyclin D1/CDK4 complex often leads to uncontrolled cell division and malignancy (23-25).

In order to further examine the mechanisms of the pro-apoptotic and antiproliferative activities of ECSB, RT-PCR and western blot analysis were performed to examine the expression of Bax, Bcl-2, cyclin D1 and CDK4. As shown in Fig. 5, ECSB significantly reduced the mRNA and protein expression of anti-apoptotic Bcl-2 as well as the pro-proliferative cyclin D1 and CDK4, whereas that of pro-apoptotic Bax was markedly increased following ECSB treatment.

In conclusion, the present study demonstrated that ECSB exhibited a potent inhibitory effect on colon cancer cell growth, which was mediated by its pro-apoptotic and antiproliferative activity. These results provide a strong scientific foundation for the development of novel anticancer agents from the bioactive ingredients in ECSB.

\section{Acknowledgements}

This study was supported by the National Natural Science Foundation of China (grant no. 81073097).

\section{References}

1. Spiro S: Lung cancer: principles and practice. Br J Cancer 85 : 1609,2001

2. Bosset JF, Calais G, Mineur L, et al: Enhanced tumorocidal effect of chemotherapy with preoperative radiotherapy for rectal cancer: preliminary results-EORTC 22921. J Clin Oncol 23 5620-5627, 2005.

3. Rougier P, Bugat R, Douillard JY, et al: Phase II study of irinotecan in the treatment of advanced colorectal cancer in chemotherapy-naive patients and patients pretreated with fluorouracil-based chemotherapy. J Clin Oncol 15: 251-260, 1997.

4. Jiang WQ, Fu FF, Li YX, et al: Molecular biomarkers of colorectal cancer: prognostic and predictive tools for clinical practice. J Zhejiang Univ Sci B 13: 663-675, 2012.

5. Hanahan D and Weinberg RA: The hallmarks of cancer. Cell 100 57-70, 2000

6. Lippman SM: The dilemma and promise of cancer chemoprevention. Nat Clin Pract Oncol 3: 523, 2006.

7. Longley DB, Allen WL and Johnston PG: Drug resistance, predictive markers and pharmacogenomics in colorectal cancer. Biochim Biophys Acta 1766: 184-196, 2006.
8. Gordaliza M: Natural products as leads to anticancer drugs. Clin Transl Oncol 9: 767-776, 2007.

9. Jia L: Cancer complementary and alternative medicine research at the US National Cancer Institute. Chin J Integr Med 18: 325-332, 2012

10. Carmady B and Smith CA: Use of Chinese medicine by cancer patients: a review of surveys. Chin Med 6: 1-8, 2011.

11. Tan KY, Liu CB, Chen AH, et al: The role of traditional Chinese medicine in colorectal cancer treatment. Tech Coloproctol 12: $1-6,2008$.

12. Chinese Pharmacopoeia Commission: Pharmacopoeia of the People's Republic of China. Vol 1. Chinese Medical Science and Technology Press, pp109-110, 2010.

13. Kim KW, Jin UH, Kim DI, et al: Antiproliferative effect of Scutellaria barbata D. Don. on cultured human uterine leiomyoma cells by down-regulation of the expression of Bcl-2 protein. Phytother Res 22: 583-590, 2008.

14. Dai ZJ, Wang XJ, Xue Q, et al: Effects of Scutellaria Barbata drug-containing serum on apoptosis and mitochondrial transmembrane potential of hepatoma $\mathrm{H} 22$ cells. Zhong Xi Yi Jie He Xue Bao 6: 821-826, 2008 (In Chinese).

15. Wei PY, Pu HQ, Wei X, Li CG and Nong S: Apoptosis-inducing effect of Scutellaria barbata extract on human lung cancer SPC-A-1 cells and the expression of apoptosis associated genes. Zhong Yao Cai 30: 1270-1273, 2007 (In Chinese).

16. Fong S, Shoemaker M, Cadaoas J, et al: Molecular mechanisms underlying selective cytotoxic activity of BZL101, an extract of Scutellaria barbata, towards breast cancer cells. Cancer Biol Ther 7: 577-586, 2008.

17. Wei L, Lin J, Wu G, et al: Scutellaria barbata D. Don induces G1/S arrest via modulation of p53 and Akt pathways in human colon carcinoma cells. Oncol Rep 29: 1623-1628, 2013.

18. Wei L, Lin J, Xu W, et al: Scutellaria barbata D. Don inhibits tumor angiogenesis via suppression of Hedgehog pathway in a mouse model of colorectal cancer. Int J Mol Sci 13: 9419-9430, 2012.

19. Adams J and Cory S: The Bcl-2 apoptotic switch in cancer development and therapy. Oncogene 26: 1324-1337, 2007.

20. Adams JM and Cory S: Bcl-2-regulated apoptosis: mechanism and therapeutic potential. Curr Opin Immunol 19: 488-496, 2007.

21. Serrano M, Hannon GJ and Beach D: A new regulatory motif in cell-cycle control causing specific inhibition of cyclin D/CDK4. Nature 366: 704-707, 1993.

22. Resnitzky D, Gossen M, Bujard H and Reed S: Acceleration of the G1/S phase transition by expression of cyclins D1 and $\mathrm{E}$ with an inducible system. Mol Cell Biol 14: 1669-1679, 1994.

23. Chung DC, Brown SB, Graeme-Cook F, et al: Overexpression of cyclin D1 occurs frequently in human pancreatic endocrine tumors. J Clin Endocrinol Metab 85: 4373-4378, 2000.

24. Kim H, Ham EK, Kim YI, et al: Overexpression of cyclin D1 and cdk4 in tumorigenesis of sporadic hepatoblastomas. Cancer Lett 131: 177-183, 1998 .

25. Keum J, Kong G, Yang S, et al: Cyclin D1 overexpression is an indicator of poor prognosis in resectable non-small cell lung cancer. Br J Cancer 81: 127-132, 1999. 